\begin{tabular}{|c|c|c|c|c|c|c|c|}
\hline \multirow{2}{*}{1905} & \multirow{2}{*}{$\begin{array}{c}\text { M. Z. } \\
\text { O-Gyalla }\end{array}$} & \multicolumn{3}{|c|}{ Vergleichstern } & \multirow{2}{*}{ Mittel } & \multirow{2}{*}{ Luft } & \multirow{2}{*}{ Bemerk. } \\
\hline & & $7^{\mathrm{n}} \cdot 74$ & $7^{\mathrm{n}} \cdot 20$ & $6^{\mathrm{min}} \cdot 90$ & & & \\
\hline März I 2 & $8^{\mathrm{h}} 20^{\mathrm{m}}$ & $8^{m} \cdot 57$ & $8^{m} \cdot 53$ & - & $8 \stackrel{m}{m} 5$ & 4 & \\
\hline 20 & 86 & $8.3^{2}$ & 8.30 & $8 \mathbf{m}_{29}$ & $8.3^{\circ}$ & 3 & 2 \\
\hline 20 & 856 & 8.46 & $8.5^{8}$ & 8.47 & $8.5^{\circ}$ & 3 & \\
\hline 25 & 937 & 8.43 & 8.39 & - & 8.41 & 2 & \\
\hline
\end{tabular}

\begin{tabular}{|c|c|c|c|c|c|c|c|}
\hline \multirow{2}{*}{1905} & \multirow{2}{*}{$\begin{array}{c}\text { M. Z. } \\
\text { O-Gyalla }\end{array}$} & \multicolumn{3}{|c|}{ Vergleichstern } & \multirow{2}{*}{ Mittel } & \multirow{2}{*}{ Luft } & \multirow{2}{*}{ Bemerk. } \\
\hline & & $7^{m} \cdot 74$ & $7^{\mathrm{m}} \cdot 20$ & $6^{\mathrm{m}} \cdot 9 \mathrm{o}$ & & & \\
\hline 29 & $9^{\mathrm{h}} 13^{\mathrm{m}}$ & $8 \cdot 57$ & $8 \cdot 54$ & - & $8 \cdot 5^{6}$ & 2 & \\
\hline ril 4 & 9 I 2 & 8.05 & 8.04 & - & 8.04 & - & $\mathrm{Ne}$ \\
\hline 5 & 940 & 8.04 & 8.14 & - & 8.09 & - & Nebeli \\
\hline 8 & 917 & 8.34 & 8.34 & - & 8.34 & 4 & \\
\hline
\end{tabular}

Bemerkungen: I. Unruhige Bilder. - 2. Heller Mondschein. - 3. Beob. durch Wolken unterbrochen.

Die Beobachtungen zeigen einen deutlichen Gang der Helligkeit des Sterns BD. $+54^{\circ} 49$, insbesondere die vom 2 I. Januar, an welchem Tage seine Helligkeit, während einer Dauer von ca. fünf Stunden, von $8^{\mathrm{m}} \cdot 5$ bis $8^{\mathrm{m}} . \circ$ zunahm und wieder bis $8.6-8 \cdot 7$ herabsank. Die Beobachtungen vom 22 . Januar lassen eine konstante Helligkeit erkennen. Leider konnte ich den Stern bis jetzt in keinem längeren Zeitraum verfolgen und der tiefe Stand des Sterns in letzterer Zeit war den Beobachtungen sehr ungünstig. Obwohl aus meinen Beobachtungen sich der definitive Charakter des Lichtwechsels des Sterns var. r90.I904 noch nicht ableiten läßt, kaṇn die Veränderlichkeit doch als zweifellos konstatiert gelten.

Osservazioni della cometa 1905 II (1904 e)

fatte a Padova.

\begin{tabular}{|c|c|c|c|c|c|c|c|c|c|c|c|c|c|c|c|c|c|c|c|}
\hline \multirow{2}{*}{$\frac{1904-05}{\text { Dic. } 3 \mathrm{I}}$} & \multicolumn{3}{|c|}{ T.m.Padova } & \multicolumn{2}{|c|}{$\Delta a$} & \multicolumn{2}{|c|}{$\Delta \delta$} & \multirow{2}{*}{$\frac{\text { Cf. }}{10.10}$} & \multicolumn{3}{|c|}{$\alpha$ app. } & \multirow{2}{*}{$\frac{\log p . \Delta}{8.777}$} & \multicolumn{3}{|c|}{$\delta$ app. } & \multirow{2}{*}{$\frac{\log p \cdot A}{0.849}$} & \multicolumn{2}{|c|}{ Red. ad 1. app. } & \\
\hline & & & & $-0^{m}$ & 385.28 & $+3^{\prime}$ & A & & & $16^{\mathrm{m}}$ & . & & -8 & 80 & & & & & \\
\hline & 6 & 10 & 35 & -0 & 22.54 & & & 6.- & I & I 7 & I 5.98 & $8.63^{8}$ & & - & & & -0.14 & -9.2 & \\
\hline $\mathbf{x}$ & 6 & 28 & 14 & & - & +7 & 49.2 & -.10 & & - & - & - & -7 & 739 & 21.9 & 845 & -0.14 & -9.2 & \\
\hline 3 & & 35 & 36 & -5 & 6.86 & +4 & & 10.10 & $\mathbf{I}$ & I9 & 5 & & -6 & I & & & & & \\
\hline 5 & & (2) & 55 & $+\mathrm{I}$ & 45.06 & +2 & 12 & ro & I & 22 & $4^{8}$ & & -4 & 423 & & & & & \\
\hline 6 & 6 & $2 \mathrm{I}$ & $5 \mathrm{I}$ & -0 & I 6.27 & +6 & 9. & 10.10 & $\mathrm{I}$ & 24 & I 4. & & -3 & 335 & 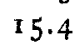 & 20 & 一 & -8.3 & \\
\hline 6 & & 26 & 8 & -2 & 59.25 & +4 & 6. & ro. Io & $\mathbf{r}$ & 24 & 17.66 & & -3 & 333 & & 820 & -0.10 & -8.4 & \\
\hline 7 & & I I & 57 & -0 & $44 \cdot 37$ & +2 & 22.5 & I0.10 & I & 25 & 41.90 & $8.120_{n}$ & -2 & 247 & $6 . \mathrm{x}$ & 4 & -0.12 & -8.2 & \\
\hline 7 & 7 & 35 & 37 & +4 & 30 & -2 & 88 & 10.10 & $\mathbf{I}$ & 25 & 4 & & -2 & 244 & I 7 & & & -8.0 & \\
\hline 7 & 10 & I 2 & I 3 & -3 & 9 & $+o$ & & 0 & $\mathbf{I}$ & 25 & 5 & & -2 & 239 & & & & & \\
\hline 8 & 6 & 5 & 30 & +0 & $25 \cdot 5^{8}$ & -2 & & 10.10 & $\mathbf{I}$ & 27 & 1 & & $-I$ & I 58 & & & & & \\
\hline 9 & & 15 & 12 & +0 & 6 & +5 & 17 & 10.10 & $I$ & 28 & & & -1 & 110 & & & & & \\
\hline 9 & & I 7 & 29 & +1 & 40 & -4 & $5^{2}$ & I0.10 & I & 28 & 4 & & $-I$ & 8 & & 2 & - & 7 & \\
\hline 9 & 9 & $4 \mathrm{I}$ & 22 & -0 & 0 & $+\mathbf{I}$ & 40 & 10.10 & $\mathbf{I}$ & 28 & & & $-I$ & 3 & & & I & -7.8 & \\
\hline 10 & & 15 & & -0 & $4 I$ & +0 & I 7 & 10.10 & $I$ & 30 & I & & -0 & 22 & 40.0 & & & $-7 \cdot 7$ & \\
\hline 10 & 7 & 4 & 30 & +2 & $\mathbf{1} 4.86$ & +0 & 48. & IO & $\mathbf{I}$ & 30 & & & -0 & $0.2 \mathrm{I}$ & & & & -7.6 & \\
\hline 10 & & 23 & 42 & +o & 48.05 & $-\mathbf{I}$ & & IO & I & & 3 & & -0 & 16 & 2 & & & -7.6 & \\
\hline I & 6 & 23 & 34 & +1 & 59.83 & -2 & 27 & 10.10 & $\mathbf{I}$ & $3^{I}$ & 5 & & +0 & 25 & & & & 4 & \\
\hline I & & 19 & 2 & -2 & 40.84 & -0 & & IO. IO & I & 31 & & & +0 & 27 & & & & -7.6 & \\
\hline I 1 & 10 & 9 & 36 & +4 & 3.02 & +0 & $5^{8 .}$ & 10.10 & $\mathbf{I}$ & $3^{2}$ & 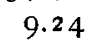 & & +0 & $3^{2}$ & & & & $-7 \cdot 3$ & \\
\hline 23 & 6 & 59 & 18 & -0 & 23.35 & -0 & 4. & 0.10 & I & 53 & & & +9 & 937 & & & & -5.8 & \\
\hline 24 & 7 & 10 & 9 & $-I$ & $4 \cdot 7$ & -I & 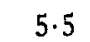 & I0.10 & I & 55 & & & +10 & 021 & & & & -5.7 & \\
\hline 2 & 7 & 8 & 35 & -0 & 48.27 & +10 & 16 & I 0.5 & $\mathbf{I}$ & & $5^{8.53}$ & & $+I I$ & 5 & & & & & \\
\hline 2 & & 43 & 18 & - I & I0. & -5 & 35 & & I & & & & +11 & 6 & & & & & \\
\hline 2 & 6 & 47 & 10 & +0 & I 0.2 & $+\mathrm{I}$ & & 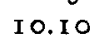 & I & & & & I I & 47 & & & & & \\
\hline 26 & 8 & 2 & 5 & $+I$ & & -3 & 36 & 0 & I & & 1.04 & & +11 & I 49 & & & & -5.4 & \\
\hline 27 & & 24 & 54 & +0 & 33 & -3 & 17 & 0.10 & 2 & I & & & $+r_{2}$ & 234 & & & 5 & $-5 \cdot 3$ & \\
\hline 28 & 6 & 55 & 47 & -2 & 44. & $-I$ & 23 & 0.10 & 2 & 2 & $58.2 x$ & & $+x 3$ & $3 \times 2$ & & & & -5.2 & \\
\hline 29 & 6 & 49 & 50 & +o & $3^{1}$. & -2 & 53 & IO.IO & 2 & 5 & 0.0 & & +13 & 354 & & & & -5.0 & \\
\hline 3 & 6 & 44 & $3^{6}$ & $+\mathrm{I}$ & 59. & $+I$ & 26 & & 2 & 7 & & & +14 & 435 & & & & & \\
\hline 30 & 7 & 57 & 36 & +1 & $5^{2}$ & -4 & & 0 & 2 & 7 & & & 14 & $4 \quad 37$ & & & & -4.8 & \\
\hline bb. 3 & 7 & I 5 & 28 & +1 & 44. & +6 & & & 2 & & & & +17 & $\begin{array}{ll}78 \\
\end{array}$ & & & & -4.5 & \\
\hline 3 & 8 & I I & $4 \mathrm{I}$ & +3 & 47 & -0 & 38.6 & Io.Io & 2 & & & & +17 & 720 & 2.0 & & & -4.4 & \\
\hline 4 & 8 & 29 & 28 & +0 & 38.87 & $+I$ & 29.1 & 10.10 & 2 & I 8 & 2. 19 & & +17 & 759 & 51.0 & & -0.04 & -4.2 & \\
\hline & 7 & 26 & 43 & +2 & 46.65 & -0 & $4 \mathrm{I} \cdot 4$ & 10.10 & 2 & & I 1.02 & 9.360 & +18 & $8 \quad 37$ & I I. 3 & 0.626 & -0.04 & -4.2 & \\
\hline
\end{tabular}




\begin{tabular}{|c|c|c|c|c|c|c|c|c|c|c|}
\hline 1905 & T.m.Padova & $\Delta \alpha$ & $\triangle 1 \delta$ & Cf. & $\alpha$ app. & $\log p . \Delta$ & $\delta$ app. & $\log p . \Delta$ & Red. ad 1. app. & * \\
\hline ebb. 6 & $7^{\mathrm{h}} 12^{\mathrm{m}} 3^{8^{\mathrm{s}}}$ & $+0^{\mathrm{ml}} 23^{\mathrm{s} .80}$ & $+1^{\prime} 50^{\prime \prime} 8$ & 10.10 & $2^{\mathrm{h}} 22^{\mathrm{mm}} 25^{\mathrm{s}} \cdot 24$ & 9.322 & $+19^{\circ} 15^{\prime} 19^{\prime \prime} 8$ & 0.611 & $-0.03-4^{\prime \prime} .2$ & \\
\hline 7 & $6543^{8}$ & -o 29.96 & $-0 \quad 29.2$ & 10.10 & $224 \quad 40.64$ & 9.263 & +I9 5257.0 & 0.595 & $-0.02-4.1$ & \\
\hline 8 & $793^{8}$ & $-052.4 \mathrm{I}$ & - I 46.5 & IO. IO & $\begin{array}{lll}2 & 27 & 0.94\end{array}$ & $9 \cdot 326$ & $+203 I$ & 0.594 & $-0.02-4.0$ & \\
\hline 9 & 7435 & to 9.44 & +3 1 1.8 & 10.10 & $22920.6 \mathrm{I}$ & 9.317 & +2 I 84.5 & 0.584 & $\begin{array}{lll}-0.02 & -3.9\end{array}$ & \\
\hline 10 & $720 \mathrm{II}$ & -048.01 & +056.1 & 10.10 & $23^{1} 44.12$ & 9.374 & $+2 \mathrm{I} 45$ II. 5 & $0.5^{85}$ & $-0.02-3.9$ & \\
\hline
\end{tabular}

Stelle di confronto.

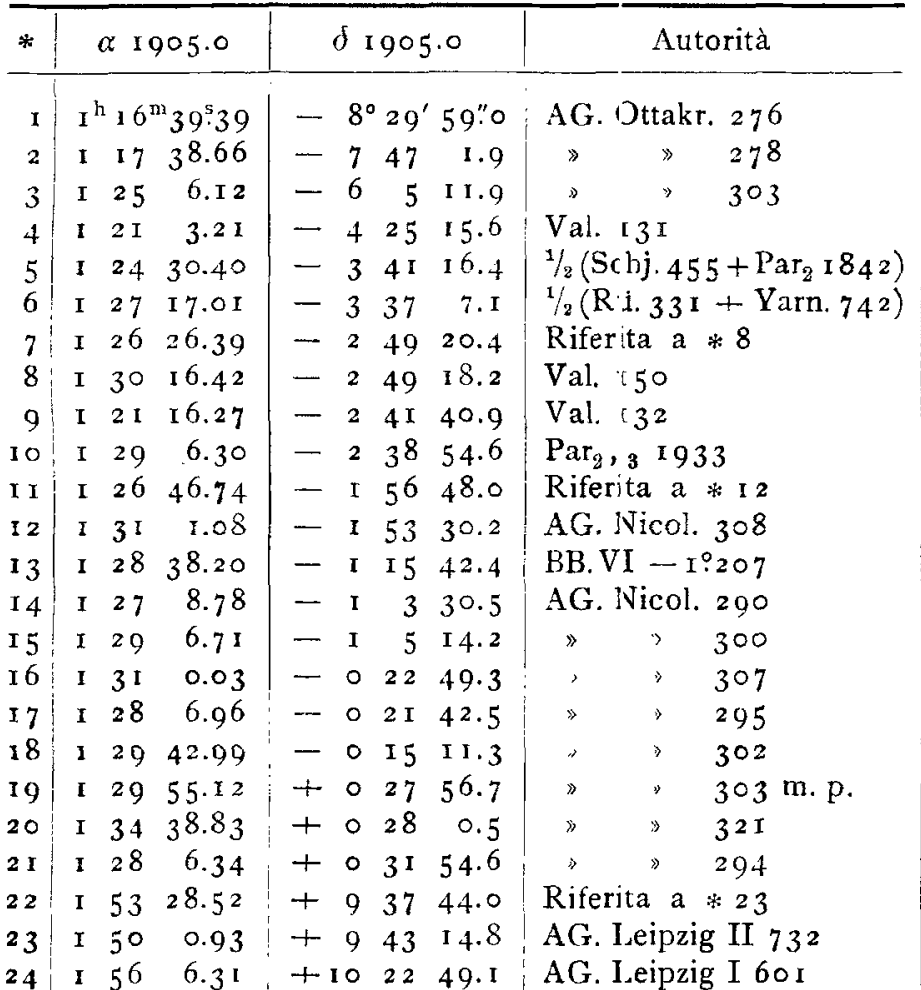

\begin{tabular}{|c|c|c|c|c|c|c|c|}
\hline$*$ & \multicolumn{3}{|c|}{$\alpha 1905.0$} & \multicolumn{2}{|c|}{$\delta$ I $9 \circ 5.0$} & \multicolumn{2}{|r|}{ Autorità } \\
\hline 25 & & 57 & ${ }^{n} 46: 85$ & $+10^{\circ} 54^{\prime}$ & $52 " 3$ & $\mathrm{AG}$ & eipzig I 609 \\
\hline 26 & $\mathbf{x}$ & & $\mathrm{I} 2.30$ & + I I I I & 47.0 & $\gg$ & $\gg 6 \times 3$ \\
\hline 27 & I & $5^{8}$ & 45.06 & + I I 45 & 55.6 & $>$ & 6 I 4 \\
\hline 28 & & $5^{8}$ & 0.22 & + I I 53 & 23.0 & $\therefore$ & 612 \\
\hline 29 & 2 & 0 & 35.53 & +1238 & $7 \cdot 5$ & $\gg$ & " 622 \\
\hline 30 & 2 & 5 & 42.79 & $+I_{3} I_{4}$ & 14.0 & 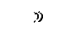 & $\gg 644$ \\
\hline 3 I & 2 & 4 & 29.31 & $+\mathrm{I}_{3} 57$ & 27.0 & $\Rightarrow$ & $» 635$ \\
\hline $3^{2}$ & 2 & 5 & 5.92 & +1434 & 23.7 & $»$ & 642 \\
\hline 33 & 2 & 5 & 19.44 & +1442 & 10.5 & $\triangleright$ & 643 \\
\hline 34 & 2 & 13 & 59.15 & +1712 & 22.3 & $\mathrm{AG}$ & erlin A 643 \\
\hline 35 & 2 & 12 & 0.59 & +1720 & 45.8 & $"$ & * $\quad \geqslant 636$ \\
\hline $3^{6}$ & 2 & 17 & $23 \cdot 3^{6}$ & $+175^{8}$ & $26 . x$ & $"$ & $" 655$ \\
\hline 37 & 2 & $x 7$ & 24.4 I & +1837 & $5^{6.9}$ & » & $\Rightarrow 656$ \\
\hline 38 & 2 & 22 & 1.47 & +1913 & 33.2 & Rife & $a \mathrm{a} * 39$ e a $* 40$ \\
\hline 39 & 2 & 20 & $2 \times \cdot 34$ & +197 & 30.7 & AG. & erlin A $67^{\circ}$ \\
\hline 40 & 2 & $2 I$ & 39.08 & +1926 & $14 \cdot 5$ & $»$ & $\Rightarrow 675$ \\
\hline 41 & 2 & 25 & 10.62 & +1053 & $3^{0.3}$ & $\gg$ & $\gg 685$ \\
\hline 42 & 2 & 27 & $53 \cdot 37$ & +2032 & 54.0 & $\gg$ & 772 \\
\hline 43 & 2 & 29 & I 1.19 & $+2 I \quad 4$ & $5^{6.6}$ & Rife & $a \mathbf{a} * 44$ \\
\hline 44 & 2 & 26 & 58.24 & $+21 \quad 2$ & 24.4 & $\mathrm{AG}$ & erlin $B 767$ \\
\hline 45 & 2 & 32 & 32.15 & $+2 \times 44$ & I $9 \cdot 3$ & Rife & a $a * 46$ e a $* 47$ \\
\hline $4^{6}$ & 2 & 32 & 46.00 & +2 I $3^{6}$ & 33.6 & $\mathrm{AG}$ & erlin B 794 \\
\hline 47 & & 36 & 5.65 & $-1-2150$ & $15 \cdot 5$ & $»$ & $\gg 807$ \\
\hline
\end{tabular}

\begin{tabular}{|c|c|c|c|c|c|c|}
\hline$*$ & \multicolumn{3}{|c|}{$\alpha 1905.0$} & \multicolumn{2}{|c|}{$\delta$ I 905.0} & Autorità \\
\hline 25 & & 57 & $n_{4} 6: 85$ & $+10^{\circ} 54^{\prime}$ & $52 " 3$ & AG. Leipzig I 609 \\
\hline 26 & $\mathbf{x}$ & & 12.30 & +II I I & 47.0 & $\gg 6 \times 3$ \\
\hline 27 & I & $5^{8}$ & 45.06 & + I I 45 & 55.6 & $\nabla 614$ \\
\hline 28 & & $5^{8}$ & 0.22 & + I I 53 & 23.0 & 612 \\
\hline 29 & 2 & $\circ$ & 35.53 & +1238 & $7 \cdot 5$ & " 622 \\
\hline 30 & 2 & 5 & 42.79 & $+I_{3}$ I 4 & 14.0 & $\gg 644$ \\
\hline $3^{I}$ & 2 & 4 & $29.3 \mathrm{I}$ & $+\mathrm{r} 357$ & 27.0 & $\geqslant 635$ \\
\hline $3^{2}$ & 2 & 5 & 5.92 & +1434 & 23.7 & $\because 642$ \\
\hline 33 & 2 & 5 & 19.44 & +1442 & 10.5 & 643 \\
\hline 34 & 2 & 13 & 59.15 & +1712 & $22 \cdot 3$ & AG. Berlin A 643 \\
\hline 35 & & 12 & 0.59 & +1720 & 45.8 & $\geqslant 636$ \\
\hline $3^{6}$ & & I7 & $23 \cdot 36$ & $+175^{8}$ & $26 . x$ & .655 \\
\hline 37 & 2 & $x_{7}$ & 24.41 & +1837 & $5^{6.9}$ & $" 656$ \\
\hline $3^{8}$ & 2 & 22 & 1.47 & +1913 & 33.2 & Riferita a $* 39$ e a $* 40$ \\
\hline 39 & 2 & 20 & $2 r \cdot 34$ & +197 & 30.7 & AG. Berlin A $67^{\circ}$ \\
\hline 40 & 2 & 21 & 39.08 & +1926 & $14 \cdot 5$ & $\Rightarrow 675$ \\
\hline 41 & 2 & 25 & 10.62 & +1953 & $3^{0.3}$ & $》 685$ \\
\hline 42 & 2 & 27 & $53 \cdot 37$ & +2032 & 54.0 & 772 \\
\hline 43 & & 29 & I 1. I 9 & $+2 I \quad 4$ & $5^{6.6}$ & Riferita a * 44 \\
\hline 44 & 2 & 26 & 58.24 & $+21 \quad 2$ & 24.4 & AG. Berlin B 767 \\
\hline 45 & 2 & 32 & 32.15 & +2144 & I $9 \cdot 3$ & Riferita a $* 46$ e a $* 47$ \\
\hline $4^{6}$ & 2 & 32 & 46.00 & +2 เ 36 & 33.6 & AG. Berlin B 794 \\
\hline 47 & & 36 & 5.65 & +2150 & $15 \cdot 5$ & $\# \gg 807$ \\
\hline
\end{tabular}

Padova, 1905 Febbrajo.

\section{A. Antoniaszi.}

\section{On the variable 47.1905 Aurigae.}

The discovery of the variability of this $\operatorname{star}(=48$ Aurigae) by Mr. T. H. Astbury of Wallingford was announced by Professor Turner in the A.N. 4010 . Observations made here fully confirm the variability of the star. The following elements of variation have been derived from observations on I 9 nights communicated by the discoverer, and Io made here by the writer:

Hove, I 905 May 12.
Maximum $=1905$ April 6.3 (J. D. 2416942.3) $+3.75 \mathrm{E}$ and these elements, although necessarily at present somewhat approximate, cannot be far from the truth.

The variation is of the $\delta$ Cephei type, the increase being considerably quicker than the decrease, the interval $M-m$ being $I_{i} I_{\text {. Th }}$. The range of variation is about $0^{m} \cdot 55$ $(5 \cdot \mathrm{m} \circ$ to $5 \cdot 55)$.

Planet Mars. (Telegramm aus Boston, Mass. vom 28. Mai.) Lowell telegraphs: Canals of Mars have been photographed here by Lampland Nilo Syrtis, Casius, Vexillum, Thoth, Cerberus, Helicon, Styx, Chaos, Liedus [Libneus ?] and others, some appearing on more than twenty negatives. Pickering.

Inhalt zu Nir. 402 I. A. Tass. Vorläufige Mitteilung der Resultate photometrischer Beobachtungen veränderlicher Sterne. I97. - A. Tass. Photometrische Beobachtungen des Veränderlichen 190.1904 Cassiopejae. 207. - A. Antoniazzi. Osservazioni della cometa 1905 II (1904 e). 209. - A. Stanley IVilliams. On the variable 47.1905 Aurigae. 21 1 . Pickering. Plartet Mars. 2 I I. 